Comment. Math. Helv. 74 (1999) 322-344

(C) 1999 Birkhäuser Verlag, Basel

\title{
Milnor link invariants and quantum 3-manifold invariants
}

\author{
Nathan Habegger* and Kent E. Orr**
}

\begin{abstract}
Let $\mathcal{Z}(M)$ be the 3-manifold invariant of Le, Murakami and Ohtsuki. We show that $\mathcal{Z}(M)=1+o(n)$, where $o(n)$ denotes terms of degree $\geq n$, if $M$ is a homology 3-sphere obtained from $S^{3}$ by surgery on an $n$-component Brunnian link whose Milnor $\bar{\mu}$-invariants of length $\leq 2 n$ vanish.

We prove a realization theorem which is a partial converse to the above theorem.

Using the Milnor filtration on links, we define a new bifiltration on the $\mathbb{Q}$ vector space with basis the set of oriented diffeomorphism classes of homology 3-spheres. This includes the Milnor level 2 filtration defined by Ohtsuki. We show that the Milnor level 2 and level 3 filtrations coincide after reindexing.
\end{abstract}

Mathematics Subject Classification (1991). 57M25, 57M99, 81Q30, $81 \mathrm{~T} 18$.

Keywords. Kontsevich Integral, Milnor link invariants, 3-manifolds Homology spheres, quantum invariants, Feynman diagrams, finite type invariants.

\section{Introduction}

The field of finite type 3-manifold invariants (also known as "perturbative quantum invariants") has developed quite rapidly over the past two years. A universal invariant of 3-manifolds, taking values in an algebra of Feynman diagrams, was introduced by Le, Murakami and Ohtsuki [LMO]. More generally, their invariant is defined for a (3-manifold, framed-link) pair. Here, universal means the invariant has been "decoupled" from Lie algebras, i.e., it involves no other choice of data.

Beginning with Ohtsuki $[\mathrm{Oh}]$, with subsequent important contributions of Garoufalidis-Levine [GL1] and Garoufalidis-Ohtsuki [GO], the study of finite-type 3 -manifold invariants culminated in the work of Le [Le]. Le showed that the LMO invariant, restricted to homology 3-spheres, was of finite type in the sense of Ohtsuki. Furthermore, in direct analogy with the universality of the Kontsevich

*This and related preprints can be obtained at http://www.math.sciences.univ-nantes.fr or http://php.indiana.edu/ korr

** Supported by a grant from the National Science Foundation, and an American Mathematical Centennial Fellowship. 
integral for the Vassiliev filtration on links in the 3-sphere (see [B2] and [LM1]), Le showed that for homology 3 -spheres, the LMO invariant is also universal in the sense that it factors any other finite type invariant.

In $[\mathrm{HM}], \mathrm{G}$. Masbaum and the first author investigated the relation between finite type link invariants and Milnor's $\bar{\mu}$-invariants of string-links. (Milnor's invariants had been shown earlier to be finite type string-link invariants [B1], [Lin].) They showed that Milnor's invariants vanish if and only if the Kontsevich integral (the universal finite type invariant of tangles) vanished in a quotient of the algebra of Feynman diagrams. This quotient is obtained by setting to zero those graphs having homology. Here the Milnor filtration of links, where a link lies in the $n^{\text {th }}$ Milnor filtration if it has vanishing Milnor invariants of length $\leq n$, corresponds to the degree of the first non-vanishing term in the "treelike" part of the Kontsevich expansion. They also proved realization theorems for Milnor's invariants and the Kontsevich integral.

This paper grew from an attempt to understand the Feynman diagrams with homology and their relation to links and 3-manifolds. It represents partial progress towards that goal. Roughly, the main idea is to fracture a Feynman diagram into contractible components. Each such diagram is the first non-vanishing part of the Kontsevich integral of some link (by the realization result of $[\mathrm{HM}]$ ). One then pieces together links and 3-manifolds realizing the given Feynman diagram in an amalgamation procedure defined in Section 7. (See in particular Theorems 7.1 and 7.3.) This interplay, between a diagram and its contractible pieces, provides a double edged sword from which vanishing results are proven as well (Theorem 6.5).

This paper is greatly indebted to the work of Le [Le]. Both conceptually and technically speaking, one may see our results as variations on the counting arguments developed in [Le] and used to prove the universality of the Le-MurakamiOhtsuki invariant. (See the Fundamental Known Results, parts (3) and (4) of Section 5.) Together with the recently discovered relationship, mentioned above, between the "treelike" part of the Kontsevich integral and Milnor's invariants, these techniques lead to more general realization and vanishing results for the $L M O$ invariant. (For applications of these ideas to the computation of the $L M O$ invariant, see also [H2], [BH], [GH].)

To express and motivate these results, we give a new bifiltration on the rational vector space of integral homology 3-spheres, defined using Milnor's filtration on links.

We arrange this paper as follows. Section 2 gives a brief description of the universal invariant of Le, Murakami and Ohtsuki, as well as a few needed and known results. Many of the ideas from sections 3 and 4 can be found in Section 5.3 of [Le]. In these sections, we establish the necessary technical tools for proving our realization and vanishing theorems. In particular, we define the notions of the variation of a link, and introduce types of Feynman diagrams together with some special lemmas about types. Section 5 defines the aforementioned bifiltration on the $\mathbb{Q}$ vector space of integral homology 3 -spheres, and discusses its relation 
to known results on previously defined filtrations. Section 6 begins the heart of the paper. Here we state and prove the vanishing result that says that the $L M O$ invariant of a certain sum of manifolds obtained via surgery, from a link with vanishing Milnor invariants of high order, will live in high degree in the corresponding algebra of Feynman diagrams. To prove this, we need what appears to be a new lemma concerning Milnor's invariants of links and proven in a separate appendix. Section 7 gives our main realization Theorems 7.1 and 7.3. These may be interpreted as a partial converse to the vanishing Theorem 6.5. Some applications will appear in the forthcoming paper [HO]. The paper concludes with an appendix which reviews the definitions of Milnor's link invariants and states some needed results regarding these profound invariants (mostly folklore, but a few new). In particular, many of the known results about Milnor's invariants work equally well for links in homology spheres, but have not appeared in this form.

\section{The universal finite type invariant}

This section provides a brief review of the universal finite type 3-manifold invariant, and some known results.

A tangle in a manifold $M$ is a smooth compact 1-manifold $X$, and a smooth embedding $T:(X, \partial X) \rightarrow\left(M^{3}, \partial M^{3}\right)$, transverse to the boundary. If $X=\coprod_{i=1}^{\ell} I_{i}$, $M=D^{2} \times I$, and the smooth embedding is fixed to be the obvious standard embedding on the boundary, this is called a string link (see [LD] or [HL1]). More generally, we may take $M=B$, a homology 3-ball with a fixed identification (and standard embedding) of the boundary with $\partial\left(D^{2} \times I\right)$, and we refer to such a tangle as a string link in a homology ball. A framed tangle (resp., framed string link) is a tangle (resp., string link) with a non-vanishing section of the normal bundle. (N.b., if the 1-manifold is oriented, the homotopy class of this section determines and is determined by a homotopy class of section of the frame bundle of the normal bundle. Also, for a knot in a homology sphere, an isotopy class of framing may be specified by an integer, known as the self-linking number or writhe.) Note that tangles may have empty boundary, so that links (in particular, the empty link) are special cases of tangles. By gluing a string link $\sigma \subset B$, along the boundary, with the trivial $\ell$-component string link in $D^{2} \times I$, we have the notion of the closure $\hat{\sigma}$ of $\sigma$, lying in the closure $\hat{B}$ of $B$, where $\hat{B}$ is the homology 3 -sphere $B \cup_{\partial B}\left(D^{2} \times I\right)$. Every link in a homology 3-sphere is the closure of a string link in a homology ball.

We refer the reader to [B2] [B3] for definitions and discussions of the (graded, completed) $\mathbb{Q}$-vector space $\mathcal{A}(X)$ of Feynman diagrams on the 1-manifold $X$. Elements of $\mathcal{A}(X)$ are represented by linear combinations of vertex-oriented diagrams $X \cup \Gamma$, subject to the AS and IHX relations. Here $\Gamma$ is a uni-trivalent graph, whose univalent vertices lie in the interior of $X$. It is customary to refer to the trivalent 
vertices of $\Gamma$ as internal vertices and to the univalent vertices of $\Gamma$ as external vertices. It is also customary to refer to the components of $X$ as solid, and to the components of $\Gamma$ as dashed. The space $\mathcal{A}(X)$ is graded by the degree, where the degree of a diagram is half the number of vertices of $\Gamma$. The degree $n$ part of $\mathcal{A}(X)$ will be denoted by $\mathcal{A}_{n}(X)$. Note that for $X=\coprod_{i=1}^{\ell} I_{i}, \mathcal{A}(X)$ has a product defined by stacking diagrams. In this case we often denote $\mathcal{A}(X)$ by $\mathcal{A}(\ell)$. An inclusion of $I$ in $X$ defines an injection of $\mathcal{A}(1)$ into $\mathcal{A}(X)$, and an action of $\mathcal{A}(1)$ on $\mathcal{A}(X)$, called connect summing. For $X=S^{1}$, such an inclusion induces an isomorphism of $\mathcal{A}(1)$ with $\mathcal{A}\left(S^{1}\right)$, which thus inherits a product structure, which is known to be abelian. For $X=\emptyset, \mathcal{A}(X)$ has a product given by disjoint union.

In [LM2], (see also [LM1]), T. Le and J. Murakami constructed an invariant $Z(T) \in \mathcal{A}(X)$, a version of the Kontsevich integral, for any framed $q$-tangle $T: X \rightarrow \mathbb{R}^{2} \times I$. (This was denoted by $\hat{Z}_{f}(T)$ in [LM2] and should not be confused with its precursor $Z_{f}(T)$. See [LM2] for the definition of $q$-structure, which technically is necessary for the definition of $Z$. However, we suppress this from the notation. In what follows, one may choose the standard $q$-structure, where needed.)

In [LMO], Le, Murakami and Ohtsuki give a 3-manifold invariant, called the LMO invariant, defined as follows:

$$
\mathcal{Z}_{n}(M)=\left(\frac{\iota_{n}(\check{Z}(L))}{\left(\iota_{n}\left(\check{Z}\left(U_{+}\right)\right)\right)^{\sigma_{+}}\left(\iota_{n}\left(\check{Z}\left(U_{-}\right)\right)\right)^{\sigma_{-}}}\right)^{(n)} \in \mathcal{A}_{n}(\emptyset) .
$$

Here $\xi^{(n)}$ denotes the degree $n$ part of $\xi \in \mathcal{A}(\emptyset) . L: \coprod_{i=1}^{\ell} S_{i}^{1} \rightarrow S^{3}$ is a framed link such that surgery on $L$ gives the 3-manifold $M$. $\check{Z}(L)=\nu^{\otimes \ell} Z(L)$ is obtained by successively taking the connected sum of $Z(L)$ with $\nu$ along each component of $L$, where $\nu$ is the value of $Z$ on the unknot with zero framing. $U_{ \pm}$is the trivial knot with positive one and negative one framing, respectively. $\sigma_{ \pm}$is the dimension of the positive and negative eigenspaces of the linking matrix for the framed link $L$.

$$
\iota_{n}: \mathcal{A}\left(\coprod_{i=1}^{\ell} S_{i}^{1}\right) \rightarrow \mathcal{A}(\emptyset)
$$

is a map, which reduces degree by $n \ell$, defined in [LMO].

We will need the following facts about the $L M O$ and Kontsevich invariants. In this paper we use the notation $o(n)$ to denote terms of degree greater than or equal to $n$.

\section{Some Useful facts}

1. Let $T$ be a framed tangle. A change of framing of \pm 1 on $T$ results in a change of $Z(T) \in \mathcal{A}(X)$ by multiplying, along the appropriate component, by $e^{ \pm \frac{\theta}{2}}$, where $\theta$ is the degree one diagram on a circle with a single dashed chord, ([LM1] Theorem 3). 
2. For an $\ell$-component string link $\sigma, Z(\hat{\sigma})$ is the image, in $\mathcal{A}\left(\coprod_{i=1}^{\ell} S_{i}^{1}\right)$, of $\nu_{\ell} Z(\sigma)$, where $\nu_{\ell} \in \mathcal{A}(\ell)$ is obtained from $\nu$ by the map induced from the projection of $\ell$ intervals to a single interval, ([LM2], Theorem 6.1). (N.b., Feynman diagrams form a contravariant homotopy functor by sending a diagram to the sum of diagrams obtained by lifting the univalent vertices lying on the target 1-manifold to their preimages.)

3. $\check{Z}\left(U_{ \pm}\right)=\nu^{2} e^{ \pm \frac{\theta}{2}}$. (This follows from the definition and the above two statements.)

4. $\nu=1+$ terms with $\geq 1$ internal vertices ([Le], Lemma 2.2).

5. $\mathcal{Z}$ is multiplicative, i.e., for integral homology 3 -spheres $M_{1}, M_{2}$, one has $\mathcal{Z}\left(M_{1} \# M_{2}\right)=\mathcal{Z}\left(M_{1}\right) \mathcal{Z}\left(M_{2}\right),[L M O]$.

6. If $T$ is any tangle, then $Z(T)=1+o(1)$. (This is easily seen for generators of the monoid of tangles, and follows from the monoidal functor property of $Z$. See [LM1].)

7. $\iota_{n}\left(\check{Z}\left(U_{ \pm}\right)\right)=(\mp 1)^{n}+o(1),[L M O]$.

\section{Variations of links}

Many of the ideas in this section can be found in Section 5.3 of [Le].

Definition 3.1. Let $\sigma$ be an $\ell$-component framed string link. For a subset $S \subset$ $\{1, \ldots, \ell\}$, let $\tau_{S}(\sigma)$ be the string link obtained by replacing the $i^{\text {th }}$ component, for each $i \in S$, with the trivial string, lying above all the other strings and having the same framing as the original. If $S=\{i\}$, then we also denote $\tau_{S}$ by $\tau_{i}$. If $\sigma^{\prime}$ is a sublink of $\sigma$, then we set $\tau_{\sigma^{\prime}}=\tau_{S}$, where $S=\left\{i \mid i\right.$ is an index of the sublink $\left.\sigma^{\prime}\right\}$.

Definition 3.2. For a framed link $L$ and a collection of indices $S$, let $\tau_{S}(L)$ be the link obtained by replacing those components with indices in $S$, by the split trivial link with the same framing. We set $\tau_{L^{\prime}}(L)=\tau_{S}(L)$, where $S=$ $\left\{i \mid i\right.$ is an index of $\left.L^{\prime} \subset L\right\}$.

Thus $\tau_{S}(\hat{\sigma})=\widehat{\tau_{S}(\sigma)}$.

Definition 3.3. Let $\Lambda \subset\{1, \ldots, \ell\}$. For an $\ell$-component framed string link $\sigma$, define the $\Lambda$ variation of $\sigma$ by,

$$
\delta_{\Lambda}(\sigma)=\prod_{i \in \Lambda}\left(1-\tau_{i}\right)(\sigma)
$$

For a link $L, \delta_{\Lambda}(L)$ is defined analogously. 
Note that for a framed link $J \cup L$, with $\Lambda=\{i \mid i$ is an index of $L\}$,

$$
\delta_{L}(J \cup L)=\prod_{i \in \Lambda}\left(1-\tau_{i}\right)(J \cup L)=\Sigma_{L^{\prime} \subset L}(-1)^{\left|L^{\prime}\right|} \tau_{L^{\prime}}(J \cup L) .
$$

For a closed 3-manifold $M,[M]$ denotes its oriented diffeomorphism class.

Definition 3.4. Given a framed link $L$ in a manifold $M$, we set

$$
[M, L]=\Sigma_{L^{\prime} \subset L}(-1)^{\left|L^{\prime}\right|}\left[M\left(L^{\prime}\right)\right] .
$$

Here $M\left(L^{\prime}\right)$ denotes the result of surgery along $L^{\prime}$. We will also use the notation

$$
[M(x)]=\Sigma_{i} x_{i}\left[M\left(L_{i}\right)\right],
$$

if $x=\Sigma_{i} x_{i} L_{i}$ is a formal linear combination of links, $L_{i} \subset M$.

We call a framed link in a homology sphere unit-framed if the self-linking numbers of each component are \pm 1 . We call a framed link admissible if it is unitframed and has vanishing pairwise linking numbers. The results below hold for unit-framed links and in particular, for admissible links.

Note that surgery on a split unit-framed unknot does not change the diffeomorphism type of the three manifold. Thus, if $L^{\prime} \subset L$, and $L \backslash L^{\prime}$ is a unit-framed link, then $\left[S^{3}\left(L^{\prime}\right)\right]=\left[S^{3}\left(\tau_{\left(L \backslash L^{\prime}\right)}(L)\right)\right]$. So for $J \subset S^{3}$ a framed link with $S^{3}(J)=M$, and $L \subset\left(S^{3} \backslash J\right) \subset M$, with $L \subset S^{3}$ unit-framed,

$$
\begin{gathered}
{[M, L]=\Sigma_{L^{\prime} \subset L}(-1)^{\left|L^{\prime}\right|}\left[M\left(L^{\prime}\right)\right]} \\
=\Sigma_{L^{\prime} \subset L}(-1)^{\left|L^{\prime}\right|}\left[S^{3}\left(J \cup L^{\prime}\right)\right] \\
=(-1)^{|L|} \Sigma_{L^{\prime} \subset L}(-1)^{\left|L \backslash L^{\prime}\right|}\left[S ^ { 3 } \left(\tau\left(L \backslash L^{\prime}\right)\right.\right. \\
=(-1)^{|L|}\left[S^{3}\left(\delta_{L}(J \cup L)\right)\right] .
\end{gathered}
$$

Lemma 3.5. Let $L \subset M$ be a framed link in a 3-manifold, and let $J \subset S^{3}$ be a link such that $S^{3}(J)=M$ and such that $L \subset\left(S^{3} \backslash J\right) \subset M$. Assume that $L \subset S^{3}$ is unit-framed. Let $m$ be the minimal number of internal vertices in each nonzero summand of $Z\left(\delta_{L_{0}}\left(J_{0} \cup L_{0}\right)\right)$, where $\left(J_{0} \cup L_{0}\right)$ is $(J \cup L)$ with zero framing on each component. Then for all $n, \iota_{n}\left(\check{Z}\left(\delta_{L}(J \cup L)\right)\right)=o\left(\frac{m}{2}\right)$. Consequently $\mathcal{Z}([M, L])=o\left(\frac{m}{2}\right)$.

Proof. Recall that $\iota_{n}$ applied to a diagram $X \cup \Gamma$, where $X$ is a disjoint union of circles, is a linear combination of trivalent diagrams $\Gamma^{\prime}$, each of which contains $\Gamma$ as a subspace. (It is obtained by gluing to $\Gamma$ certain elements $T_{n}^{m}$, and replacing 
each resulting circle component with the value $-2 n$. See [LMO].) So the number of vertices of each $\Gamma^{\prime}$ is at least the number of internal vertices of $\Gamma$. Hence the number of vertices of each non-zero term of $\iota_{n}\left(\check{Z}\left(\delta_{L}(J \cup L)\right)\right)$ is at least the number of internal vertices of each non-zero term in $\check{Z}\left(\delta_{L}(J \cup L)\right)$.

By Useful Facts 1 of Section 2, one computes $\check{Z}\left(\delta_{L}(J \cup L)\right)$ from $Z\left(\delta_{L}\left(J_{0} \cup L_{0}\right)\right)$ by connect summing with $\nu e^{n_{i} \frac{\theta}{2}}$ along the $i$-th component of $J \cup L$, whose framing is $n_{i}$. As this cannot decrease the number of internal vertices by Useful Fact 4 , this yields $\iota_{n}\left(\check{Z}\left(\delta_{L}(J \cup L)\right)\right)=o\left(\frac{m}{2}\right)$.

By equation (2) above,

$$
\mathcal{Z}([M, L])=(-1)^{|L|} \mathcal{Z}\left(S^{3}\left(\delta_{L}(J \cup L)\right)\right) .
$$

Moreover, $\mathcal{Z}_{n}\left(S^{3}\left(\delta_{L}(J \cup L)\right)\right)$ is computed from $\iota_{n}\left(\check{Z}\left(\delta_{L}(J \cup L)\right)\right)$ using equation (1). The result follows from Useful Fact 7 .

\section{Special types of Feynman diagrams}

For each $i \in\{1, \ldots, \ell\}$, we have an inclusion $j_{i}: \mathcal{A}(\ell-1) \rightarrow \mathcal{A}(\ell)$ and a projection $\epsilon_{i}: \mathcal{A}(\ell) \rightarrow \mathcal{A}(\ell)$ onto the image of $j_{i}$. Here, for a diagram $\xi=X \cup \Gamma, X=\coprod_{i=1}^{\ell} I_{i}$, representing a class in $\mathcal{A}(\ell)=\mathcal{A}\left(\coprod_{i=1}^{\ell} I_{i}\right)$, we have

$$
\epsilon_{i}(\xi)= \begin{cases}\xi & \text { if } \Gamma \text { has no vertex on the } i \text { th component } \\ 0 & \text { otherwise. }\end{cases}
$$

For an $\ell$ component string link $\sigma$, the operation $\tau_{i}$ is conjugate to the operation $\tau_{\ell}(\sigma)$, i.e., $\tau_{i}(\sigma)=\beta_{i}^{-1}\left(\tau_{\ell}\left(\beta_{i} \sigma \beta_{i}^{-1}\right)\right) \beta_{i}$, for a certain braid $\beta_{i}$ inducing the permutation $s_{i}=(\ell, \ell-1, \ldots, i)$. Thus, $Z\left(\tau_{\ell}(\sigma)\right)=\epsilon_{\ell}(Z(\sigma))$ (see [LM2], or [LMO] Proposition 1.1) and, for $i \neq \ell, Z\left(\tau_{i}(\sigma)\right)=\alpha^{\prime-1} \epsilon_{\ell} \alpha^{\prime}{ }_{i}(Z(\sigma))$, where $\alpha^{\prime}{ }_{i}$ is the operator which conjugates by $Z\left(\beta_{i}\right)=b_{i}$. Let $\alpha_{i}=\alpha^{\prime}{ }_{i} s_{i}$. Note that $s_{i}^{-1} \epsilon_{\ell} s_{i}=\epsilon_{i}$. Thus $Z\left(\tau_{i}(\sigma)\right)=\alpha_{i}^{-1} \epsilon_{i} \alpha_{i}(Z(\sigma))$.

Define $\tau_{i}: \mathcal{A}(\ell) \rightarrow \mathcal{A}(\ell)$ by $\tau_{\ell}=\epsilon_{\ell}$ and, for $i \neq \ell, \tau_{i}=\alpha_{i}^{-1} \epsilon_{i} \alpha_{i}$. Thus, by definition, we have that $Z\left(\tau_{i}(\sigma)\right)=\tau_{i}(Z(\sigma))$.

Definition 4.1. Let $X=\coprod_{i=1}^{\ell} X_{i}$.

We say a diagram $\xi=X \cup \Gamma$ is of type $\Lambda \subset\{1, \ldots, \ell\}$ if $\Gamma$ has at least one vertex on $X_{j}$, for all $j \in \Lambda$.

$\xi=X \cup \Gamma$ is said to be of degree (resp. tree, resp. loop) type $n$ if each component (resp. tree component, resp. component) of $\Gamma$ has degree (resp. degree, resp. rank of its first homology group) $\geq n$.

Lemma 4.2. Let $X=\coprod_{i=1}^{\ell} I_{i}$. Let $\xi=X \cup \Gamma, \xi=o(n)$, be of type $\Lambda$, of degree type $p$, of tree type $q$ and of loop type $r$. Let $b \in \mathcal{A}(\ell)$ be grouplike. Then 
$b \xi b^{-1}=\xi+\eta, \eta=o(n+1)$, where $\eta$ is a linear combination of terms $X \cup \Gamma^{\prime}$ of type $\Lambda$, of degree type $p$, of tree type $q$ and of loop type $r$.

Proof. In a complete Hopf algebra, grouplike elements are exponentials of primitives. Note that for $b=\exp (\beta), b \xi b^{-1}$ is given by $\Sigma_{k \geq 0} \frac{\left(a d_{\beta}\right)^{k}(\xi)}{k !}$, where $\left(a d_{\beta}\right)^{0}(x)=$ $x, a d_{\beta}(x)=\beta x-x \beta$ and $\left(a d_{\beta}\right)^{i}$ is the $i^{\text {th }}$ composition of $a d_{\beta}$ with itself. Since $\beta$ is primitive, $\beta=o(1)$ and is a linear combination of terms with connected dashed graph.

Observe that if one uses the STU relation to permute two univalent vertices on distinct dashed components, this produces a third graph with one less univalent vertex and one fewer component which contains subgraphs isomorphic to each of the original dashed components. So if we expand the commutator $\beta \xi-\xi \beta$ using the STU relation to successively permute the univalent vertices of $\Gamma$ with those of the connected dashed graph of a term in $\beta$, the result is a sum of terms each of whose connected components of their dashed graphs contain as subgraph a connected component of $\Gamma$. The result follows.

Lemma 4.3. Let $X=\coprod_{i=1}^{\ell} I_{i}$. Let $\xi_{i}=X \cup \Gamma_{i}$ be of degree type $p_{i}$, of tree type $q_{i}$ and of loop type $r_{i}$. Assume $\prod \xi_{i}$ is of type $\Lambda$ and that $\prod \xi_{i}=o(n)$. Then $\left(1-\tau_{i}\right)\left(\prod \xi_{i}\right)=\left(1-\epsilon_{i}\right)\left(\prod \xi_{i}\right)+\eta$, where $\eta=o(n+1)$ and is a linear combination of terms which are of type $(\Lambda \cup\{i\})$ and which are products $\prod \eta_{i}$ of terms $\eta_{i}$, where $\eta_{i}=X \cup \Gamma_{i}^{\prime}$ is of degree type $p_{i}$, of tree type $q_{i}$ and of loop type $r_{i}$.

Remark 4.4. Note that under the hypothesis that $\prod \xi_{i}$ is of type $\Lambda$, one has that $\left(1-\epsilon_{j}\right)\left(\prod \xi_{i}\right)= \begin{cases}\prod \xi_{i} & \text { if } \prod \xi_{i} \text { is of type }(\Lambda \cup\{j\}) \\ 0 & \text { otherwise. }\end{cases}$

Proof. For $i=\ell$ there is nothing to prove, since $\tau_{\ell}=\epsilon_{\ell}$. For $i \neq \ell$, one has that

$$
1-\tau_{i}=1-\alpha_{i}^{-1} \epsilon_{i} \alpha_{i}=\alpha_{i}^{-1}\left(1-\epsilon_{i}\right) \alpha_{i},
$$

where $\alpha_{i}$ is conjugation by a grouplike element. The lemma follows by applying Lemma 4.2 twice, using the above remark and the fact that the conjugate of a product is the product of conjugates.

Repeated application of Lemma 4.3 proves the following.

Corollary 4.5. Let $X=\coprod_{i=1}^{\ell} I_{i}$. Let $\xi_{i}=X \cup \Gamma_{i}$ be of degree type $p_{i}$, of tree type $q_{i}$ and of loop type $r_{i}$. Assume $\prod \xi_{i}=o(n)$. Then $\prod_{j \in \Lambda}\left(1-\tau_{j}\right)\left(\prod \xi_{i}\right)=$ $\prod_{j \in \Lambda}\left(1-\epsilon_{j}\right)\left(\prod \xi_{i}\right)+\eta$, where $\eta=o(n+1)$ and is a linear combination of terms which are of type $\Lambda$ and which are products $\prod \eta_{i}$ of terms $\eta_{i}=X \cup \Gamma_{i}^{\prime}$ of degree type $p_{i}$, of tree type $q_{i}$ and of loop type $r_{i}$. 
Remark 4.6. Note that $\prod_{j \in \Lambda}\left(1-\epsilon_{j}\right)\left(\prod \xi_{i}\right)= \begin{cases}\prod \xi_{i} & \text { if } \prod \xi_{i} \text { is of type } \Lambda \\ 0 & \text { otherwise. }\end{cases}$

\section{A bifiltration on the space of integral homology 3-spheres}

We designate by $\mathcal{S}$ the rational vector space with basis the set of oriented diffeomorphism classes of integral homology 3 -spheres. This is an algebra under connected sum. $\mathcal{Z}$ extends linearly to define an algebra homomorphism on $\mathcal{S}$.

Denote by $\mathcal{S}_{n}$ the universal filtration (or LMO filtration), i.e.,

$$
\mathcal{S}_{n}=\operatorname{ker}\left(\mathcal{Z}_{<n}: \mathcal{S} \rightarrow \mathcal{A}_{<n}(\emptyset)\right) .
$$

We extend the Ohtsuki filtration (see below) to a bifiltration using Milnor's filtration as follows. (See the appendix for definitions and brief discussions on Milnor's invariants.)

Recall that we say that a link in a homology 3 -sphere is admissible, if it is unitframed and the linking numbers of its 2-component sublinks vanish. A sublink of an admissible link is admissible. If $L=J \cup J^{\prime} \subset M$ is an admissible link, then $M(J)$ is a homology sphere and $J^{\prime} \subset M(J)$ is again admissible.

Definition 5.1. For a fixed $n$, define the length $r$ Milnor filtration, $\mathcal{S}_{n}^{r}$, to be the subspace of $\mathcal{S}$ generated by the elements $[M, L]$, where $L$ denotes an admissible $n$-component framed link with vanishing Milnor $\bar{\mu}$-invariants of length $\leq r$.

The length two Milnor filtration, $\mathcal{S}_{n}^{2}$, is the well known Ohtsuki filtration of $\mathcal{S}$. If $M$ is an integral homology 3-sphere, $K$ a knot, and $(L \cup K) \subset M$ is an admissible link with vanishing Milnor invariants of length $\leq r$, then the Milnor invariants of $L \subset M(K)$ vanish up to length $r$ by Lemma A.3 of the Appendix. It follows from the fundamental equation

$$
[M, L \cup K]=[M, L]-[M(K), L],
$$

that the vector spaces $\mathcal{S}_{n}^{r}$ form a bifiltration, i.e., that $\mathcal{S}_{n+1}^{r} \subset \mathcal{S}_{n}^{r}$. (That $\mathcal{S}_{n}^{r+1} \subset$ $\mathcal{S}_{n}^{r}$ is immediate.)

We now state, in approximate historical order, and using the above notation, the fundamental known results regarding the Ohtsuki filtration and its relation to the $L M O$ invariant. We recommend that the reader pause here to become familiar with these results and with the notation.

\section{Fundamental Known Results}

1. $\mathcal{S}_{3 n+1}^{2}=\mathcal{S}_{3 n+2}^{2}=\mathcal{S}_{3 n+3}^{2}$.

2. There is a surjective map $A_{n}(\emptyset) \rightarrow \frac{\mathcal{S}_{3 n}^{2}}{\mathcal{S}_{3 n+1}^{2}}$. 
3. $\mathcal{S}_{3 n+1}^{2} \subset \mathcal{S}_{n+1}$.

4. The map $\mathcal{Z}_{n}: \mathcal{S}_{3 n}^{2} \rightarrow \mathcal{A}_{n}(\emptyset)$ induces a map $\frac{\mathcal{S}_{3 n}^{2}}{\mathcal{S}_{3 n+1}^{2}} \rightarrow \mathcal{A}_{n}(\emptyset)$, which is (up to sign) a left inverse to the map in (2). Consequently, the map $\mathcal{A}_{n}(\emptyset) \rightarrow \frac{\mathcal{S}_{3 n}^{2}}{\mathcal{S}_{3 n+1}^{2}}$ in (2), and the induced map $\frac{\mathcal{S}_{3 n}^{2}}{\mathcal{S}_{3 n+1}^{2}} \rightarrow \mathcal{A}_{n}(\emptyset)$, are inverse isomorphisms.

5. $\mathcal{S}_{3 n}^{2}=\mathcal{S}_{n}$.

6. The map $\mathcal{Z}_{\leq n}: \frac{\mathcal{S}}{\mathcal{S}_{n+1}} \rightarrow \mathcal{A}_{\leq n}(\emptyset)$ is an isomorphism.

Remark 5.2. Building on $[\mathrm{Oh}],(1)$ is proven in [GL1]. Building on [Oh] and [GL1], (2) is proven in [GO]. (3) and (4) are proven in ([Le]), and (5) and (6) follow inductively from (1)-(4).

In summary, the Ohtsuki and $L M O$ filtrations agree after a shift in indexing, and the $L M O$ expansion, restricted to homology 3 -spheres, is the universal finite type invariant. Here, a finite type invariant in the sense of Ohtsuki is one which vanishes on $\mathcal{S}_{n}^{2}$, for some $n$. $\mathcal{Z}$ is universal in the sense that any such finite type invariant factors through $\mathcal{Z}_{\leq n}$, for some $n$. (More precisely, combining (1), (5), and (6) above, one has that an invariant vanishes on $\mathcal{S}_{3 n+1}^{2}=\mathcal{S}_{3 n+2}^{2}=\mathcal{S}_{3 n+3}^{2}$ if and only if the invariant factors through $\mathcal{Z}_{\leq n}$.)

\section{A vanishing theorem}

For a 1-manifold $X$, let $\mathcal{A}^{t}(X)$ be the quotient of $\mathcal{A}(X)$ obtained by considering as relations all trivalent diagrams containing a non-simply connected, dashed component. For a tangle $T$, we denote by $Z^{t}(T)$ the image of $Z(T)$ in $\mathcal{A}^{t}(X)$. In particular, for a string link $\sigma, Z^{t}(\sigma)$ lies in $A^{t}(\ell)$.

There is a coalgebra isomorphism $\mathcal{A}(\ell)$ with $\mathcal{B}(\ell)$, the Hopf algebra of $\ell$-labeled chinese characters (i.e., linear combinations, modulo $A S$ and $I H X$ relations of vertex-oriented uni-trivalent graphs, whose univalent vertices are labeled with elements of the set $\{1, \ldots, \ell\}$ ). This isomorphism sends the primitives of $\mathcal{A}(\ell)$, denoted $\mathcal{P}(\ell)$, to the primitives of $\mathcal{B}(\ell)$, denoted $\mathcal{C}(\ell)$, which is the span of the connected chinese characters.

The above isomorphism passes to a coalgebra isomorphism of $\mathcal{A}^{t}(\ell)$ with $\mathcal{B}^{t}(\ell)$, the Hopf algebra of $\ell$-labeled forests (i.e., chinese characters whose components are trees). This yields an isomorphism of the primitives of $\mathcal{A}^{t}(\ell)$, denoted $\mathcal{P}^{t}(\ell)$, with the primitives of $\mathcal{B}^{t}(\ell)$, denoted $\mathcal{C}^{t}(\ell)$, which is the span of the chinese characters which are trees.

In $[\mathrm{HM}]$, an investigation of the relationship of the Kontsevich integral and Milnor's invariants produced the following theorem.

Habegger-Masbaum Theorem. Let $L \subset S^{3}$ be a 0-framed link with vanishing 
Milnor invariants of length $\leq r+1$. Then the Kontsevich expansion, $Z(L)$, can be expressed as a linear combination of diagrams, none of which contain a dashed component which is a tree of degree $\leq r$.

Lemma 6.1 below will be combined with a slight refinement (Proposition 6.3) of the Habegger-Masbaum theorem to prove our main vanishing result, Theorem 6.5 .

From equation $(2), \mathcal{Z}([M, L])=(-1)^{|L|} \mathcal{Z}\left(S^{3}\left(\delta_{L}(J \cup L)\right)\right)$, for a framed link $J \subset S^{3}$ such that $S^{3}(J)=M$ and $L \subset\left(S^{3} \backslash J\right) \subset M$, provided $J \cup L \subset S^{3}$ is admissible. One key point here is that one has the liberty to choose $J$ conveniently. Lemma 6.1 provides such a convenient choice for $J$.

Lemma 6.1. Suppose that $M=S^{3}\left(J^{\prime}\right)$ is a homology sphere, where $J^{\prime}$ is an admissible framed link with $m$ components. Let $L \subset M$ be a link with vanishing Milnor invariants of length $\leq r$. Then there is an $m$-component link $J \subset S^{3}$, with $M=S^{3}(J)$, such that $L \subset\left(S^{3} \backslash J\right) \subset M$, and such that all Milnor invariants of length $\leq r$ vanish for $(J \cup L) \subset S^{3}$.

Lemma 6.1 is proven in the Appendix on Milnor's invariants, and has the following corollary.

Corollary 6.2. $\mathcal{S}_{n}^{r}$ is the subspace $\mathcal{S}_{n}^{r}\left(S^{3}\right)$ of $\mathcal{S}_{n}$ spanned by the elements $\left[S^{3}, L\right]$, where $L$ is an admissible m-component framed link (i.e., trivial pairwise linking numbers and framing \pm 1 on each component), with $m \geq n$, whose Milnor $\bar{\mu}$ invariants of length $\leq r$ vanish.

Proof. Clearly $\mathcal{S}_{n}^{r}\left(S^{3}\right) \subset \mathcal{S}_{n}^{r}$.

Let $[M, L]$ be a generator of $\mathcal{S}_{n}^{r}$, i.e., L is an admissible framed link with $\geq n$ components and vanishing Milnor invariants of length $\leq r$. By Lemma 6.1, $M=S^{3}(J)$ for some admissible framed link $J$, such that $J \cup L \subset S^{3}$ has vanishing Milnor invariants of length $\leq r$. The proof that $[M, L] \in \mathcal{S}_{n}^{r}\left(S^{3}\right)$ proceeds by induction on $|J|$, the number of components of $J$.

The result holds for $|J|=1$ by Lemma A.3 of the Appendix, since in this case, we have the fundamental equation (3) of Section 5 ,

$$
[M, L]=\left[S^{3}(J), L\right]=\left[S^{3}, L\right]-\left[S^{3}, J \cup L\right] \in \mathcal{S}_{n}^{r}\left(S^{3}\right) .
$$

Assume the result for $|J|<n$. Let $J=J^{\prime} \cup K, K$ a knot. Then, using the fundamental equation, the inductive hypothesis, and Lemma A.3, we have

$$
\left[S^{3}(J), L\right]=\left[\left(S^{3}\left(J^{\prime}\right)\right)(K), L\right]=\left[S^{3}\left(J^{\prime}\right), L\right]-\left[S^{3}\left(J^{\prime}\right), K \cup L\right] \in \mathcal{S}_{n}^{r}\left(S^{3}\right),
$$

which proves the inductive step.

The following is the promised refinement of the Habegger-Masbaum Theorem. 
Proposition 6.3. Let $r \geq 2$. Let $J \cup L$ be a 0 -framed link in $S^{3}$ with vanishing Milnor invariants of length $\leq r$. Then $Z\left(\delta_{L}(J \cup L)\right)$ has the following properties. 1. $Z\left(\delta_{L}(J \cup L)\right)$ can be expressed as a linear combination of diagrams, none of which contain a dashed component which is a tree of degree $<r$, and

2. each such diagram has at least two vertices on each component of $L$.

Proof. Let $\sigma_{J} \cup \sigma_{L}$ be a string link such that $\sigma_{J} \widehat{\cup} \sigma_{L}=J \cup L$. By $[\mathrm{HM}], Z_{<r}^{t}\left(\sigma_{J} \cup\right.$ $\left.\sigma_{L}\right)=1$. It follows (as in [HM], see also the proof of Theorem 7.1 below) that $Z\left(\sigma_{J} \cup \sigma_{L}\right)-1$ is of tree type $r . Z\left(\delta_{L}(J \cup L)\right)$ is the image, in $\mathcal{A}\left(\coprod_{i=1}^{\ell} S_{i}^{1}\right)$, of the result of multiplying $Z\left(\delta_{\sigma_{L}}\left(\sigma_{J} \cup \sigma_{L}\right)\right)$ by $\nu_{\ell}$ (Useful Fact 2). Since $\nu_{\ell}$ is a sum of diagrams without trees, part (1) of the Proposition follows from Corollary 4.5, which also gives that each diagram has at least one vertex on each component of $L$.

Since $r \geq 2$, there are no dashed components without internal vertices. It follows that each non-zero diagram must have at least two vertices on each component of $L$, since the $S T U$ relation implies that a term with only one vertex on a solid circle, which is not the vertex of a chord, must be zero. (To see this, push an internal vertex next to a solid circle to the solid circle using the $S T U$ relation. The result is the difference of 2 terms, each having 2 vertices on the circle. As these vertices may be interchanged, up to isotopy, the 2 terms are the same and hence their difference is zero.)

For a real number $n$, let $[n]$ denote the greatest integer $\leq n$.

Lemma 6.4. Let $\Gamma$ be a uni-trivalent graph. Suppose $\Gamma$ has at least $2 n$ external vertices, and that each tree component has at least $2 r$ vertices. Then $\Gamma$ has at least

$$
2 n-2\left[\frac{2 n}{r+1}\right]-1
$$

internal vertices.

Proof. By an Euler characteristic counting argument, every tree with $2 s$ vertices has $s+1$ external vertices and $s-1$ internal vertices, and thus 2 fewer internal vertices than external vertices. Every component which is not a tree has at least as many internal vertices as external vertices.

Let $k$ be the number of tree components. If $k \leq\left[\frac{2 n}{r+1}\right]$, then since by hypothesis there are at least $2 n$ external vertices, there are at least $2 n-2 k \geq 2 n-2\left[\frac{2 n}{r+1}\right]$ internal vertices. Suppose $k>\left[\frac{2 n}{r+1}\right]$. Then since each tree component has at least $r-1$ internal vertices, one has that there are at least

$$
k(r-1) \geq\left(\left[\frac{2 n}{r+1}\right]+1\right)(r-1) \geq 2 n-2\left[\frac{2 n}{r+1}\right]-1
$$

internal vertices. 
Theorem 6.5 is the main theorem of this section.

Theorem 6.5. Let $r \geq 2$. Let $L \subset M$ be an admissible $n$-component link with vanishing Milnor $\bar{\mu}$-invariants of length $\leq r$. Then

$$
\mathcal{Z}([M, L])=o\left(n-\left[\frac{2 n}{r+1}\right]\right) .
$$

That is,

$$
\mathcal{S}_{n}^{r} \subset \mathcal{S}_{n-\left[\frac{2 n}{r+1}\right]}
$$

In particular, $\mathcal{S}_{n}^{2 n} \subset S_{n}$.

Proof. By Lemma 6.1 above, there is a link $J \subset S^{3}$ with $M=S^{3}(J)$, such that $L \subset\left(S^{3} \backslash J\right) \subset M$ and such that all Milnor invariants of length $\leq r$ vanish for $J \cup L \subset S^{3}$. (Here, $J \cup L$ has unit-framing and $J_{0} \cup L_{0}$ denotes the same link but with zero framing.) By Proposition 6.3, each term in the expansion $Z\left(\delta_{L}\left(J_{0} \cup L_{0}\right)\right)$ has at least two vertices on each component of $L$, no dashed component of which is a tree of degree $<r$. By Lemma 6.4, each summand has at least $2 n-2\left[\frac{2 n}{r+1}\right]-1$ internal vertices. The result follows from Lemma 3.5.

Remark 6.6. For $r=2$, Theorem 6.5 is the Fundamental Result (3) of Section 5. For $r=3$, this gives $\mathcal{S}_{2 n}^{3} \subset \mathcal{S}_{n}$. Using Fundamental Result (5), the inclusion $\mathcal{S}_{2 n}^{3} \subset \mathcal{S}_{n}$ is equivalent to the main theorem of [H] , which was proven geometrically, without mention of the $L M O$ invariant.

\section{Realization results}

In this section we prove a realization theorem which is a partial converse to the vanishing Theorem 6.5.

Suppose a collection of $k$ connected uni-trivalent graphs, $\Gamma_{i}$, has a total of $2 \ell$ univalent vertices labeled with elements of the set $\{1, \ldots, \ell\}$, where each of the $\ell$ labels occurs exactly twice. The amalgam of the graphs $\Gamma_{i}$ is the trivalent graph obtained by joining the univalent vertices in pairs according to their labels.

We define the amalgamation map

$$
A: \mathcal{P}_{n_{1}}^{t}(\ell) \times \ldots \times \mathcal{P}_{n_{k}}^{t}(\ell)=\mathcal{C}_{n_{1}}^{t}(\ell) \times \ldots \times \mathcal{C}_{n_{k}}^{t}(\ell) \rightarrow \mathcal{A}_{n}(\emptyset)
$$

to be the map which sends collections of chinese characters which are trees to the amalgam of these trees if together they have exactly 2 univalent vertices of each label, and sends the collection to zero otherwise. One has that $2 \ell-k=\Sigma n_{i}$, and that $n=\ell+k$.

In the following theorem, we use the symbol 1 to denote both the trivial string link and $1 \in \mathcal{A}_{0}(X)$. The usage should be clear from context. 
Theorem 7.1. Let $\xi=A\left(\xi_{1}, \ldots, \xi_{k}\right) \in \mathcal{A}_{n}(\emptyset)$ be the amalgam of the primitive elements $\xi_{i} \in \mathcal{A}_{n_{i}}^{t}(\ell)$. Let $\sigma_{i} \subset D^{2} \times I$ be $\ell$-component zero framed string links such that $Z^{t}\left(\sigma_{i}\right)=1+\xi_{i}+o\left(\left|n_{i}\right|+1\right)$. Assume in addition that $Z\left(\sigma_{i}\right)=1+o\left(n_{i}-m+1\right)$, where $m$ is the minimum of the $n_{i}$. Then

$$
\mathcal{Z}\left(\left[S^{3},\left(\prod \widehat{\left(\sigma_{i}-1\right)}{ }_{+1}\right]\right)=\xi+o(n+1),\right.
$$

where $L_{+1}$ denotes the link $L$, with positive one framing on each component.

Remark 7.2. This theorem is a generalization of the case (considered by Le) where the $\sigma_{i}$ are split unions of a trivial string link and a Borromean string link. This special case was used to prove Fundamental Result (4). Note that if all the $n_{i}$ are the same, the condition $Z\left(\sigma_{i}\right)=1+o\left(n_{i}-m+1\right)$ is automatically satisfied. In particular, we have the following special case.

Theorem 7.3. Let $\hat{\sigma} \subset S^{3}$ be the closure of an $\ell$-component 0 -framed string link $\sigma$. Let $\hat{\sigma}_{+1}$ denote the same link, but with +1 framing on every component. Suppose the Milnor invariants of length $\leq 2 \ell-2$ of $\hat{\sigma}$ vanish. Then $\mathcal{Z}\left(\left[S^{3}, \hat{\sigma}_{+1}\right]\right)=o(\ell-1)$ and

$$
\mathcal{Z}_{\ell-1}\left(\left[S^{3}, \hat{\sigma}_{+1}\right]\right)=A\left(Z_{2 \ell-1}^{t}(\sigma)\right),
$$

where $A: \mathcal{P}_{2 \ell-1}^{t}(\ell)=\mathcal{C}_{2 \ell-1}^{t}(\ell) \rightarrow \mathcal{A}_{\ell-1}(\emptyset)$ is the amalgamation map described above.

Remark 7.4. The hypothesis of vanishing Milnor invariants implies that, $Z^{t}(\sigma)=$ $1+o(2 \ell-1)$ and hence that $Z_{2 \ell-1}^{t}(\sigma)$ is primitive (see [HM], Remark 2.2). Moreover, in $[\mathrm{HM}], Z_{2 \ell-1}^{t}(\sigma)$ was identified with Milnor's invariants of length $2 \ell$ of $\sigma$.

Proof of Theorem 7.1. We set $X=\coprod_{i=1}^{\ell} I_{i}$ and $\Lambda=\{1, \ldots, \ell\}$.

We claim that

$$
Z\left(\delta_{\Lambda} \prod\left(\sigma_{i}-1\right)\right)=\prod_{j \in \Lambda}\left(1-\epsilon_{j}\right)\left(\prod \xi_{i}\right)+\eta,
$$

where $\eta$ consists of a linear combination of terms of type $\Lambda$ which are products $\prod \eta_{i}$ of terms $\eta_{i}=X \cup \Gamma_{i}$, where $\Gamma_{i}$ is of degree type $n_{i}-m+1$ and tree type $n_{i}$, and such that not every $\Gamma_{i}$ is a tree of degree $n_{i}$. To see this, note that (see $[\mathrm{HM}])$, one has that $Z\left(\sigma_{i}\right)=\exp \left(\xi_{i}^{t}+\xi_{i}^{h}\right)$, where $\xi_{i}^{t}=\xi_{i}+o\left(n_{i}+1\right)$ is a linear combination of diagrams whose dashed graphs are trees, and $\xi_{i}^{h}=o\left(n_{i}-m+1\right)$ is a linear combination of diagrams whose dashed graphs are connected, none of which is a tree. It follows that $Z\left(\sigma_{i}\right)=\xi_{i}+\eta_{i}$, where $\eta_{i}$ is a linear combination of terms of degree type $n_{i}-m+1$ and tree type $n_{i}$, none of which has dashed graph a tree of degree $n_{i}$.

Since $Z\left(\delta_{\Lambda} \prod\left(\sigma_{i}-1\right)\right)=\delta_{\Lambda}\left(\prod\left(Z\left(\sigma_{i}\right)-1\right)\right)$, with $\delta_{\Lambda}=\prod_{j \in \Lambda}\left(1-\tau_{j}\right)$, the claim is a direct application of Corollary 4.5. 
By Useful Fact 2 and the definition of $\check{Z}$, one obtains $\check{Z}\left(\delta_{\Lambda}\left(\left(\prod\left(\widehat{\sigma_{i}}-1\right)\right)\right)\right.$ by multiplying $Z\left(\delta_{\Lambda} \prod\left(\sigma_{i}-1\right)\right)$ by the element $\nu^{\otimes \ell} \nu_{\ell}$ to get a new element of $\mathcal{A}(\ell)$ and taking the image in $\mathcal{A}\left(\coprod_{i=1}^{\ell} S_{i}^{1}\right)$. Note that $\nu^{\otimes \ell} \nu_{\ell}=1+o(1)$ and is a sum of terms without trees by Useful Fact 4 . Thus $\check{Z}\left(\delta_{\Lambda}\left(\left(\prod\left(\widehat{\sigma_{i}}-1\right)\right)\right)\right.$ is the image in $\mathcal{A}\left(\coprod_{i=1}^{\ell} S_{i}^{1}\right)$ of

$$
\prod_{j \in \Lambda}\left(1-\epsilon_{j}\right)\left(\prod \xi_{i}\right)+\eta^{\prime}
$$

where $\eta^{\prime}$ satisfies the same conditions as $\eta$.

By the same argument used in Proposition 6.3, the map to $\mathcal{A}\left(\coprod_{i=1}^{\ell} S_{i}^{1}\right)$ kills any term in the above expression with just one vertex on some component. Thus we may eliminate each term in the above expression which does not have at least 2 vertices on every component. Note that the expression $\prod_{j \in \Lambda}\left(1-\epsilon_{j}\right)\left(\prod \xi_{i}\right)$ is the sum of those terms in $\prod \xi_{i}$ with at least one vertex on each component. Let $\pi\left(\prod \xi_{i}\right)$ be the sum of those terms in $\prod \xi_{i}$ with at least two vertices on each component. Thus we have that $\check{Z}\left(\delta_{\Lambda}\left(\left(\prod\left(\widehat{\sigma_{i}}-1\right)\right)\right)\right.$ is the image in $\mathcal{A}\left(\coprod_{i=1}^{\ell} S_{i}^{1}\right)$ of

$$
\pi\left(\prod \xi_{i}\right)+\eta^{\prime \prime},
$$

where $\eta^{\prime \prime}$ satisfies the same conditions as $\eta$ and each term $\prod \eta_{i}$ in $\eta^{\prime \prime}$ has at least 2 vertices on every component.

Note that any term in $\pi\left(\prod \xi_{i}\right)$ has exactly 2 vertices on each component, and exactly $2 n$ internal vertices, since it is a linear combination of products of $k$ terms with degree $n_{1}, \ldots, n_{k}$, whose dashed components are trees. We claim that the terms $\prod \eta_{i}$ in $\eta^{\prime \prime}$ have strictly more than $2 n$ internal vertices. To see this, note that since any such term has at least $2 \ell$ external vertices, if it had $\leq 2 n=2 \ell-2 k$ internal vertices, it's dashed graph would have to contain at least $k$ trees (see the proof of 6.4). Let $I$ be the collection of indices in $\{1, \ldots, k\}$ for which $\Gamma_{i}$ does not contain a tree. Note that if $I=\emptyset$, then each $\Gamma_{i}$ contains a tree which contributes at least $n_{i}-1$ internal vertices and if $\Gamma_{i}$ has exactly $n_{i}-1$ internal vertices, then it is a tree of degree $n_{i}$. Since not all the $\Gamma_{i}$ are trees of degree $n_{i}$, the total number of internal vertices is $>\Sigma\left(n_{i}-1\right)=2 n$ in this case. Thus we may assume that $I \neq \emptyset$. For all $i$ in $I$, we have that the degree of $\Gamma_{i}$ is $\geq n_{i}-m+1$, and since $\Gamma_{i}$ does not contain a tree, $\Gamma_{i}$ contributes at least $n_{i}-m+1$ internal vertices. For each index $i$ not in $I, \Gamma_{i}$ contains at least one tree which contributes at least $n_{i}-1$ internal vertices. This accounts for $k-|I|$ trees. The remaining $|I|$ trees must have at least $m-1$ internal vertices each. Thus the total number of internal vertices is at least $\Sigma_{i \in\{1, \ldots, k\} \backslash I}\left(n_{i}-1\right)+\Sigma_{i \in I}\left(\left(n_{i}-m+1\right)+(m-1)|I|>2 n\right.$.

Thus we have shown that any term in $\check{Z}\left(\delta_{\Lambda}\left(\left(\prod\left(\widehat{\sigma_{i}}-1\right)\right)\right)\right.$, other than those in $\pi\left(\prod \xi_{i}\right)$, must have strictly more internal vertices than $2 n$. It follows from equations (1) and $(2)$ that $\mathcal{Z}\left(\left[S^{3},\left(\prod \widehat{\left(\sigma_{i}\right.}-1\right)_{+1}\right]\right)=o(n)$ and that

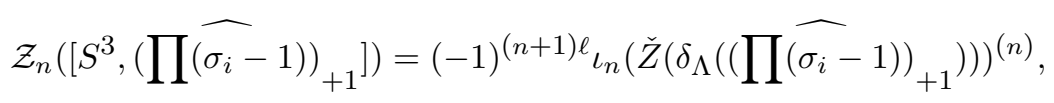


(since by Useful Fact (7) of Section 2, the denominator in equation (1) satisfies $\left.\left(\iota_{n}\left(\check{Z}\left(U_{+}\right)\right)\right)^{\ell}=(-1)^{n \ell}+o(1)\right)$.

To compute $\check{Z}\left(\delta_{\Lambda}\left(\left(\prod\left(\widehat{\sigma_{i}}-1\right)\right)_{+1}\right)\right)$, we multiply $\check{Z}\left(\delta_{\Lambda}\left(\left(\prod\left(\widehat{\sigma_{i}}-1\right)\right)\right)\right)$ by $e^{\frac{\theta}{2}}$, along each component. Proceeding as in Section 5 of [Le], since $\iota_{n}$ decreases degree by $n \ell$ and annihilates any summand with $<2 n$ vertices on any component, and since the terms in $\pi\left(\prod \xi_{i}\right)$ each have two vertices on each component of $\coprod_{i=1}^{\ell} I_{i}$, the only terms having $\geq 2 n$ vertices on each component in degree $n(\ell+1)$ are obtained by multiplying $\pi\left(\prod \xi_{i}\right)$ and the degree $n-1$ term in each exponential, $e^{\frac{\theta}{2}}$. Thus we have

$$
\mathcal{Z}_{n}\left(\left[S^{3},\left(\prod \widehat{\left(\sigma_{i}-1\right)}\right)+1\right]\right)=(-1)^{(n+1) \ell} \iota_{n}\left(\left(2^{n-1}(n-1) !\right)^{-\ell} \gamma\right),
$$

where $\gamma$ consists of $\pi\left(\prod \xi_{i}\right)$ together with $n-1$ trivial chords added to each solid component of $\coprod_{i=1}^{\ell} I_{i}$. Applying $\iota_{n}$ replaces each pair of external vertices of a term in $\pi\left(\prod \xi_{i}\right)$ by a dashed chord connecting them, and multiplies by $\left((-2)^{n-1}(n-\right.$ $1) !)^{\ell}$. (This follows from the definition of $\iota_{n}$ in [LMO], as the element $T_{n}^{2 n}$ consists of $n$ dashed chords and all resulting circle components are replaced by $-2 n$. See also Lemma 5.4 of [Le].) This yields $\xi$, proving the theorem.

The following Theorems are application of the results in this paper. They will appear with proof along with other applications in [HO].

The following application of Theorem 7.3 is a slight refinement of a result announced in [Le], which states that a degree $n$ primitive can be realized as the first non-vanishing term of surgery on an $n$-component link.

Theorem 7.5. (Compare with [Le].) Let $\xi \in \mathcal{A}_{n}(\emptyset)$ be primitive and represented by a connected graph $\Gamma$. Then there is a knot $K \subset S^{3}$ with +1 framing, such that

$$
\mathcal{Z}\left(S^{3}(K)\right)=1+(-1)^{n+1} \xi+o(n+1)
$$

There are numerous questions that naturally arise regarding our bifiltration on the space of homology three spheres. (See Definition 5.1.) Recall that after reindexing, the Ohtsuki and LMO filtrations agree, i.e, $\mathcal{S}_{3 n}^{2}=\mathcal{S}_{n}$ (see the Fundamental Result (5)). The following is also true.

Theorem 7.6. $\mathcal{S}_{2 n}^{3}=\mathcal{S}_{n}$

Thus the $L M O$, Ohtsuki and length 3 Milnor filtration all agree after indexing changes.

We conclude the paper with another question regarding our bifiltration that is suggested by the vanishing Theorem 6.5 . 
Question. Are any of the inclusions $\mathcal{S}_{n}^{\partial} \subset \mathcal{S}_{n}^{\infty} \subset \cap_{N} \mathcal{S}_{n}^{N} \subset \mathcal{S}_{n}^{2 n+r+1} \subset \mathcal{S}_{n}^{2 n+r} \subset$ $\mathcal{S}_{n}$ equalities, $r \geq 0$ ? In particular, is $\mathcal{S}_{n}^{2 n}=\mathcal{S}_{n}$ ?*

\section{Appendix A. Milnor's invariants}

This appendix is divided into two sections. The first gives a brief account of Milnor's $\bar{\mu}$-invariants for string links in homology balls and links in homology spheres. Although some of this is new to the literature, it is all folklore, and the proofs are only outlined. The second section contains some new results that were needed in this paper.

\section{A.1. Definitions and folklore}

Let $\sigma \subset B^{3}$ be a string link of $\ell$ components in a homology 3-ball. By connecting a collection of meridional circles to the base point by pairwise disjoint paths, one gets a homology equivalence $\phi: \vee^{\ell} S^{1} \rightarrow E_{\sigma}$, where $E_{\sigma}$ is the exterior of $\sigma$. Let $F=F(\ell)$ be the free group on $\ell$ generators, the fundamental group of $\vee^{\ell} S^{1}$, and let $\pi$ be the fundamental group of $E_{\sigma}$. Then by Stalling's theorem [St], $\phi$ induces an isomorphism

$$
\frac{F}{F_{k}} \rightarrow \frac{\pi}{\pi_{k}}
$$

for all positive integers $k$. Here, $G_{k}$ is the $k^{\text {th }}$ lower central subgroup of the group $G$ defined by $G_{1}=G$, and inductively $G_{k+1}=\left[G, G_{k}\right]$ is the group of commutators of $G$ and $G_{k}$.

The link exterior of $\hat{\sigma}$ is a union of the string link exterior $E_{\sigma}$, with a trivial string link exterior. By Van Kampen's theorem, we get the following presentation due to Milnor [M1], where $F_{k}$ means the $k$ fold commutators in the generators $x_{1}, \ldots, x_{\ell}$, and $\lambda_{i}$ is a word in the generators representing the $i^{t h}$ longitude of the link $\bmod \pi_{k}$.

$$
\frac{\pi}{\pi_{k}} \cong\left\{x_{1}, \ldots, x_{\ell} \mid\left[x_{i}, \lambda_{i}\right], i=1, \ldots, \ell, F_{k}\right\}
$$

To define the length $k$ Milnor $\bar{\mu}$-invariant, $\bar{\mu}\left(\iota_{1}, \ldots, \iota_{k}\right)$, of the string link $\sigma$, choose $n \geq k$ and consider the quotient $\frac{\pi}{\pi_{n}}$ in the meridional generators. Then $\bar{\mu}\left(\iota_{1}, \ldots, \iota_{k}\right)$ is the coefficient of $t_{\iota_{1}} \cdots t_{\iota_{k-1}}$ in the Magnus expansion of $\lambda_{\iota_{k}}$ (see [M1], [M2] and [MKS]).

As an immediate application of Magnus' work on the lower central series of the free group, Milnor observes that the longitudes of $\sigma$ are trivial in $\frac{\pi}{\pi_{k}}$ if and only if all $\bar{\mu}$-invariants for $\sigma$ of length $\leq k$ vanish.

* The equality $\mathcal{S}_{n}^{\partial}=\mathcal{S}_{n}$, has been recently proven by Gussarov. 
For a link $L \subset M^{3}$, in a homology 3-sphere $M^{3}$, the Milnor $\bar{\mu}$-invariants are defined to be the corresponding $\bar{\mu}$-invariants of any string link $\sigma$ such that $\hat{\sigma}=L$. These integers depend on the choice of the string link $\sigma$ with $\hat{\sigma}=L$ but are independent of these choices in a quotient of the integers depending only on the values of Milnor invariants of lower length. In particular, the invariants of first nonvanishing degree are well defined integer valued invariants (see [M1] and [M2]).

An easy argument using the Magnus expansion proves the following well known lemma, whose proof we omit.

Lemma A.1. All Milnor invariants of a link $L \subset M$ of length $\leq k$ vanish if and only if $\frac{F}{F_{n}} \cong \frac{\pi}{\pi_{n}}$ for $n \leq k+1$.

Definition A.2. In the above case, we say $\pi_{1}(M \backslash L)$ has free nilpotent quotients of length $\leq k+1$.

Lemma A.3. Let $(L \cup J) \subset M$ be an admissible framed link in a homology sphere $M$, and let $L^{\prime} \subset M(L)$ be the dual link (i.e., $L^{\prime}$ is the link for which surgery undoes surgery on $L)$. Suppose all $\bar{\mu}$-invariants of length $\leq r$ vanish for $L \cup J$. Then all $\bar{\mu}$-invariants of length $\leq r$ vanish for $L^{\prime} \cup J \subset M(L)$ (and in particular for any sublink of $L^{\prime} \cup J$, e.g. $\left.J\right)$.

Proof. As observed in Lemma A.1, the $\bar{\mu}$-invariants of length $\leq r$ vanish for a link if and only if the fundamental group of the link complement has free nilpotent quotients of length $\leq r+1$. In particular, this is a function of the fundamental group of the complement of the link alone.

Lemma A.4. If $L^{\prime} \subset M$ is a link in a homology 3-sphere with vanishing Milnor invariants of length $\leq r-1$, then there is a link $L \subset S^{3}$ with the same Milnor invariants of length $\leq r$.

Proof. In [O], it was shown that the links in $S^{3}$ with vanishing Milnor invariants of length $\leq k-1$, modulo equivalence if they possess the same length $k$ invariants, form a free abelian group of rank $m N_{k}-N_{k+1}$, where $m$ is the number of components, and $N_{k}$ is the rank of $\frac{F_{k}}{F_{k+1}}$. This computed the number of (linearly independent) Milnor invariants of a given length. In [HL], this group was identified with a free abelian group $\mathcal{K}$, where $\mathcal{K}$ is the kernel of the homomorphism

$$
\oplus \frac{F_{k}}{F_{k+1}} \rightarrow \frac{F_{k+1}}{F_{k+2}}
$$

which sends $\left(\lambda_{1}, \ldots, \lambda_{m}\right) \mapsto \prod\left[x_{i}, \lambda_{i}\right]$.

To see that any element in $\mathcal{K}$ is realized, one uses an Orr-type construction (see $[\mathrm{HL}])$. To see that the longitudes always lie in $\mathcal{K}$, one represents the link as the closure of a string link. After removing a neighborhood of the string link, one has 
a 3-manifold with boundary a closed surface. The top cell of this surface gives the needed relation. This latter argument also works for links in any homology sphere showing the longitudes lie in $\mathcal{K}$, proving the result.

Remark A.5. In fact, links in a fixed homology sphere realize exactly the same Milnor invariants as links in a sphere. To see this, just note that any link in the sphere lies in a ball and hence can be considered as lying in the given homology sphere.

\section{A.2. Some Lemmas}

Given a multi-index $I$, we denote the multiplicity of $i$ in $I$ by $m_{i}(I)$. Given a uni-trivalent graph $\Gamma$ with vertices labeled by integers, we denote the multiplicity of the integer $i$ in this graph by $m_{i}(\Gamma)$.

The following proposition makes extensive use of the realization techniques via integration of links developed by Cochran [C1]. It is a straightforward generalization of a result due to Cochran, [C2]. For a link $L$ and a multi-index $I=\left(n_{1}, \ldots, n_{k}\right)$, we denote $\bar{\mu}\left(n_{1}, \ldots, n_{k}\right)$ by $\mu_{L}(I)$.

Proposition A.6. Let $L \subset S^{3}$ be an $\ell$-component link whose Milnor invariants of length $\leq r-1$ vanish. Let $m_{i} \in \mathbb{N}, i=1, \ldots, \ell$, be such that $\Sigma m_{i}=r$. Let $\Lambda=\left\{i \mid m_{i} \neq 0\right\}$. Then there is a string link $\sigma=\sigma_{1} \cup \ldots \cup \sigma_{\ell}$ such that

1. the sub-string link $\sigma^{\prime}=\cup_{i \in \Lambda} \sigma_{i}$ is a Brunnian string link,

2. the string link $\sigma-\sigma^{\prime}$ is a split trivial sublink of $\sigma$,

3. $\bar{\mu}_{\sigma}(I)= \begin{cases}\bar{\mu}_{L}(I) & \text { if } m_{i}(I)=m_{i}, \text { for all } i \\ 0 & \text { otherwise. }\end{cases}$

In particular, the individual components of $\sigma$ are unknotted.

Proof. According to Cochran [C1], any Milnor invariant $\bar{\mu}(I)$ is a linear combination of equivalency classes of "linkings", $\lambda_{\Gamma}$, i.e. $\bar{\mu}(I)=\Sigma n_{\Gamma}^{I} \lambda_{\Gamma}$. Here the $\Gamma$ are simply connected, uni-trivalent graphs (i.e., trees), whose univalent vertices are labeled with exactly those indices occurring in $I$, and with the same multiplicities, i.e., $m_{i}(I)=m_{i}(\Gamma)$ for all $i$. The higher order linking number functions $\lambda_{\Gamma}$ and the coefficients $n_{\Gamma}^{I}$ do not depend on the link $L$.

Let $a_{I}=\bar{\mu}_{L}(I)$. Then the system of equations $a_{I}=\Sigma n_{\Gamma}^{I} \ell_{\Gamma}$ has an integral solution in the variables $\ell_{\Gamma}$ given by the values $\lambda_{\Gamma}(L)$ of the higher order linkings of the link $L$.

Consider the system of equations in the variables $\ell_{\Gamma}$

$$
\begin{aligned}
0 & =\Sigma n_{\Gamma}^{I} \ell_{\Gamma} \text { for all } I \text { such that } m_{i}(I) \neq m_{i} \text { for some } i \\
a_{I} & =\Sigma n_{\Gamma}^{I} \ell_{\Gamma} \text { for all } I \text { such that } m_{i}(I)=m_{i} \text { for all } i .
\end{aligned}
$$

Notice that this system of equations has an integral solution given by $\ell_{\Gamma}=\lambda_{\Gamma}(L)$ if $m_{i}(\Gamma)=m_{i}$ for all $i$, and $\ell_{\Gamma}=0$ if $m_{i}(\Gamma) \neq m_{i}$ for some $i$. 
Using such solutions, Cochran shows in [C2], pages 652-3, how to construct a link realizing the above solution and satisfying the desired conditions.

Specifically, Cochran constructs certain links $B_{\Gamma} \subset S^{3}$ obtained by Bing doubling the Hopf link, (or the Whitehead link, if $\Gamma$ admits an order 2 symmetry), connecting the components with the same label and adding trivial components if necessary. This link is either Brunnian or the split union of a Brunnian link and a trivial link, and may be taken to be the closure of a string link with the same properties. These links come equipped with a system of Seifert surfaces for which $\lambda_{\Gamma^{\prime}}\left(B_{\Gamma}\right)=1$, if $\Gamma=\Gamma^{\prime}$, and 0 otherwise. Thus $\bar{\mu}_{I}\left(B_{\Gamma}\right)=0$, if for some $i$, $m_{i}(I) \neq m_{i}(\Gamma)$.

Cochran shows that there is a linear combination $\Sigma n_{\Gamma} B_{\Gamma}$ with the same lowest order Milnor invariants as $L$. The subsum taken over all equivalency classes of trees, $\Gamma$, with $m_{i}(\Gamma)=m_{i}$ for all $i$, yields a link with the desired properties. Replacing link by string link, and connected sum by product of string links, yields the stated result.

1) holds since a product of Brunnian string links is Brunnian. 2) holds since no nontrivial linkings are associated to indexing sets $I$ with $m_{i}(I)=0$. 3) holds by the additivity of the first non-vanishing Milnor invariants under products of string links.

Proposition A.6 has a number of interesting corollaries.

Corollary A.7. Let $L \cup K \subset S^{3}$ be a link with vanishing Milnor invariants of length $\leq r-1$, where $K \subset S^{3}$ is a knot and where $L$ has vanishing Milnor invariants of length $\leq r$. Then there is a string link $\sigma \cup \sigma^{\prime}$, whose closure has Milnor invariants which agree with those of $L \cup K$ up to length $\leq r$, where $\sigma$ is a trivial string link and $\sigma^{\prime}$ is a trivial 1-component string link.

Proof. Since all $\bar{\mu}$-invariants of $L$ of length $\leq r$ vanish, if $I$ is any multi-index such that $\bar{\mu}_{I}(L \cup K) \neq 0$, then $m_{\ell}(I) \neq 0$, where $\ell$ is the index of the knot $K$. Take the product of the string links given in Proposition A.6 corresponding to those collections $m_{i} \in \mathbb{N}, i=1, \ldots, \ell$, such that $\Sigma m_{i}=r$ and such that $m_{\ell} \neq 0$. This yields a string link with the same Milnor invariants as $L \cup K$ by the additivity of the first non-vanishing Milnor invariants. Every term in this product is the split union of a Brunnian string link containing the component corresponding to $K$, and a trivial string link. Removing the component corresponding to $K$ thus gives a trivial string link by condition 1) of Proposition A.6 and, moreover, the component corresponding to $K$ is trivial.

Proposition A.8. Let $K \subset M$ be a knot in a homology sphere $M$. Let $L \cup K \subset M$ be a link such that all Milnor $\bar{\mu}$-invariants of $L$ of length $\leq r$ vanish. Then $K$ is isotopic in $M$ to a knot $K^{\prime}$ such that $L \cup K^{\prime}$ has vanishing Milnor invariants of length $\leq r$. 
Proof. Clearly the result holds for $r=2$, since linking numbers may be changed locally by changing crossings.

Assume inductively it holds for $r-1$, where $r>2$. Thus we may assume that $L \cup K$ has vanishing Milnor invariants up to length $r-1$.

Changing the orientation of $L$ and taking the mirror image gives a link whose first non-vanishing Milnor invariants are the negatives of the Milnor invariants of $L \cup K$. Thus, by Lemma A.4 and Corollary A.7, we can find a string link $\sigma \cup \sigma^{\prime}$ whose Milnor invariants are the negatives of those of $L \cup K$, and such that $\sigma$ and $\sigma^{\prime}$ are trivial. By additivity of the first non-vanishing Milnor invariants, the closure of the product of $\sigma \cup \sigma^{\prime}$ with a string link version of $L \cup K$ has vanishing Milnor invariants of length $\leq r$. Since both $\sigma$ and $\sigma^{\prime}$ are trivial, this modification leaves the link $L$ and the knot $K$ unchanged, up to isotopy.

Proof of Lemma 6.1. Let $M=M^{\prime}\left(K^{\prime}\right)$ for some knot $K^{\prime} \subset M^{\prime}$, and let $L \subset M$ be a link with vanishing Milnor invariants of length $\leq r$. Let $K^{\prime \prime}$ denote the dual of $K^{\prime}$. By Proposition A.8, $K^{\prime \prime}$ is isotopic in $M$ to a knot $K^{\prime \prime \prime}$, where $L \cup K^{\prime \prime \prime}$ has vanishing Milnor invariants of length $\leq r$. Since $K^{\prime \prime \prime}$ is isotopic to $K^{\prime \prime}$ in $M$, $M\left(K^{\prime \prime \prime}\right)=M^{\prime}$. Let $K$ be the dual of $K^{\prime \prime \prime}$. Then $M^{\prime}(K)=M$. By Lemma A.3, $L \cup K \subset M^{\prime}$ has vanishing Milnor invariants of length $\leq r$. Taking $M^{\prime}=S^{3}$, this also proves the lemma for the case $m=1$.

Assume inductively the case $m-1$. If $M=S^{3}\left(J^{\prime}\right)$, with $J^{\prime}$ admissible, $J^{\prime}=$ $K^{\prime} \cup J^{\prime \prime}, K^{\prime}$ a knot, set $M^{\prime}=S^{3}\left(J^{\prime \prime}\right)$. Then $M=M^{\prime}\left(K^{\prime}\right)$. By the previous paragraph, there is $K \subset M^{\prime}$ such that $M^{\prime}(K)=M$, with $L \cup K \subset M^{\prime}$ having vanishing Milnor invariants of length $\leq r$. Applying the inductive hypothesis to $L \cup K \subset M^{\prime}$ yields a link $J^{\prime \prime \prime} \subset S^{3}$ with $m-1$ components such that $L \cup K \cup J^{\prime \prime \prime} \subset$ $S^{3}$ has vanishing Milnor invariants of length $\leq r$, and $S^{3}\left(J^{\prime \prime \prime}\right)=M^{\prime}$. Then $J=K \cup J^{\prime \prime \prime}$ satisfies the lemma, since $S^{3}\left(K \cup J^{\prime \prime \prime}\right)=S^{3}\left(J^{\prime \prime \prime}\right)(K)=M^{\prime}(K)=M$.

\section{Acknowledgments}

The authors wish to thank Tim Cochran for his suggestions for improving parts of the appendix.

This work began during a visit of the second author to the University of Nantes during June of 1996. He thanks the Department of Mathematics for the friendly and productive environment they provided for this research, and the University of Nantes for its support. We both thank the Mathematical Sciences Research Institute in Berkeley, California, where the work continued. The first author wishes to thank the CNRS for its support, and the University of Nantes for delegating his time towards research during the academic year 96-97. Lastly, the second author extends his thanks to the American Mathematical Society and its members for the support provided by the AMS Centennial Fellowship. 


\section{References}

[B1] D. Bar-Natan, Vassiliev homotopy string link invariants, Journal of Knot Theory and its Ramifications (1995), 13-32.

[B2] D. Bar-Natan, On the Vassiliev knot invariants, Topology 34 (1995), 423-472.

[B3] D. Bar-Natan, Non-Associative tangles. In: Geometric Topology (W. Kazez, Ed.), Proc. Georgia Int. Topology Conf. 1993, AMS/IP Studies in Advanced Mathematics, 1997.

[BH] A. Beliakova, N. Habegger, The Casson-Walker-Lescop Invariant as a Quantum 3-manifold Invariant, (preprint, July 1997).

[C1] T. Cochran, Derivatives of links, Milnor's concordance invariants and Massey products, AMS Memoirs 84(427) (1990).

[C2] T. Cochran, k-Cobordism for links in $\mathbf{S}^{3}$, Trans. AMS Vol. 327 2, Oct.1991, 641-654.

[GH] S. Garoufalidis, N. Habegger, The Alexander Polynomial and Finite Type 3-manifold Invariants, (preprint, July 1997).

[GL1] S. Garoufalidis, J. Levine, On finite type 3-manifold invariants II, (preprint, June 1995).

[GL2] S. Garoufalidis, J. Levine, Finite type 3-manifold invariants, the Mapping Class Group, and Blinks, (preprint, March 1996).

[GO] S. Garoufalidis, T.Ohtsuki, On finite type 3-manifold invariants III: Manifold Weight Systems, (preprint, August 1995).

[H1] N. Habegger, Finite type 3-manifold invariants: a proof of a conjecture of Garoufalidis, (preprint, July 1995).

[H2] N. Habegger, A Computation of the Universal Quantum 3-manifold Invariant for Manifolds of Rank Greater than 2, (preprint, December 1996).

[HL1] N. Habegger and X. S. Lin, The classification of Links up to Homotopy, Journal of the A.M.S. Vol. 3 No. 2 (1990), 389-419.

[HL2] N. Habegger and X. S. Lin, On link concordance and Milnor's $\bar{\mu}$-invariants, (preprint, January 1997).

[HM] N. Habegger, G. Masbaum, The Kontsevich Integral and Milnor's Invariants, (preprint, April 1997).

[HO] N. Habegger, Kent E. Orr, Applications of Existence and Vanishing Theorems for Finite Type Three Manifold Invariants, (preprint, October 1998).

[LD] J. Y. Le Dimet, Cobordisme d'Enlacement de Disques, Bull. Soc. Math. France 32 (1988), 116.

[Le] T. Le, An invariant of integral homology 3-spheres which is universal for all finite type invariants, to appear in "Novikov's seminar II".

[LM1] T. Le and J. Murakami, The universal Vassiliev-Kontsevich invariant for framed oriented links, Compositio Math. 102 (1996), 41-64.

[LM2] T. Le and J. Murakami, Parallel version of the universal Vassiliev-Kontsevich integral, (preprint, 1995), to appear in J. Pure and Appl. Algebra.

[LM3] T. Le and J. Murakami, Representations of the category of tangles by Kontsevich's iterated integral, Commun.Math. Phys. 168 535-562.

[LMO] T. Le, J. Murakami and T. Ohtsuki, On a universal quantum invariant of 3-manifolds, preprint, q-alg/9512002, 1995.

[Lin] X. S. Lin, Power series expansions and invariants of links, Geometric Topology (W. Kazez, Ed.), Proc. Georgia Int. Topology Conf. 1993, AMS/IP Studies in Advanced Mathematics, 1997.

[MKS] W. Magnus, A. Karrass, and D. Solitar, Combinatorial Group Theory, Pure and Appl. Math. vol XIII, Interscience, New York 1996.

[M1] J. Milnor, Link groups, Annals of Math. 59 (1954), 177-195.

[M2] J. Milnor, Isotopy of links, Algebraic Geometry and Topology, Princeton Univ. Press. Princeton, NJ 1957.

[Oh] T. Ohtsuki, Finite type invariants of integral homology 3-spheres, to appear in Jour. of 
Knot Theory and its Rami..

[O] K. E. Orr, Homotopy invariants of links, Invent. Math. 95(2) (1989), 379-394.

[St] J. Stallings, Homotopy and Central Series of Groups, J. Algebra (1965), 170-181.

Nathan Habegger

UMR 6629 CNRS

Université de Nantes

Department de Mathematiques

2 rue de la Houssiniere

F-44072 NANTES Cedex 03

France

e-mail: habegger@math.univ-nantes.fr
Kent E. Orr

Department of Mathematics Indiana University

Bloomington, IN 47405

USA

e-mail: korr@ucs.indiana.edu

(Received: October 23, 1998) 